\title{
TEORÍA DE LA MENTE EN LA ESQUIZOFRENIA
}

Ana Guinea Hidalgo

Javler Tirapu Ustárroz

Milagros Pollán Rufo

Servicio de Neuropsicología y Neuropsiquiatria. Clinica Ubarmin del Servicio Navarro de Selud

\section{RESUMEN}

A las alteraciones cognitivas descritas en la esquizofrenia, se ha venido a sumar en los últimos años la afectación de la denominada «teoria de le mentey, entendida como la capacidad de atribuir estados mentales y emocionales en uno mismo y en otros.

Siguiendo estas líneas de investigación el presente estudio tiene por objeto evaluar esta capacidad en pacientes del espectro de la esquizofrenia. Para ello se han recopilado las principales pruebas utilizadas clásicamente para este fin, y que recogerian en su conjunto la complejidad del constructo y los diferentes componentes que comprende. La muestra está compuesta de 40 sujetos; 20 pacientes diagnosticados de trastornos del espectro de la esquizofrenia y 20 sujetos control equiparados según edad y nivel educativo. A todos los sujetos se les aplican distintas pruebas de evaluación de la teorla de la mente: creencias de primer y segundo orden; historias extrañas de Happé; faux pas test; test de la mirada; dilema moral; prueba de reconocimiento de expresiones faciales. Además al grupo

Correspondencla: Unidad de Psico-Oncologla. Hospital Duran I Reynals. Avd. Gran Via sin. km 2,7. 08907 L'Hospitalet. Barcelona. Teléfono: 933357 011-ext. 3717. Fax. 932607783. Correo electrónico: gcosta@ico.scs.es.

Este estudio forma parte del proyecto que lleva por título Adaptación Psicologica y Morbilidad Psiquiátrica en Pacientes con Cáncer financiado por la Agència d'Avaluació de Tecnologia i Recerca Mediques (N Proyecto: 102/19/2004). 
de pacientes se les aplica la escala PANSS, para valorar el estado psicopatológico en el momento de la evaluación.

Los resultados muestran diferencias significativas entre ambos grupos en algunas de las pruebas aplicadas, tanto en pruebas de procesamiento cognitivo como en pruebas en las que es necesario un procesamiento emocional de la información: Creencias de segundo orden; Faux Pas; test de la mirada y la prueba de reconocimiento de expresiones faciales.

Son muchas las investigaciones que se centran en cómo los déficit en esta capacidad metarrepresentacional determinan el nivel de adaptación social. En el caso de la esquizofrenia el deterioro funcional y social podría ser explicado en gran parte por estos problemas en la atribución de estados mentales $y$ especialmente emocionales.

Palabras clave: ESQUIZOFRENIA; TEORIA DE LA MENTE, COGNICION SOCIAL; CREENCIAS DE PRIMER Y SEGUNDO ORDEN; FAUX PAS; HISTORIAS EXTRAN̂AS DE HAPPE; TEST DE LA MIRADA; DILEMA MORAL.

\section{SUMMARY}

To the cognitive alterations described in the schizophrenia, it has been added in the last years, the affectation of so called "theory of mind", understood as the capacity to attribute mental and emotional states in one itself and in others.

Following these lines of investigation the present study has evaluated this capacity in patients of the schizophrenia disorder spectrum through classic principal tests.

The sample is composed of 40 subjects; 20 patients diagnosed of the schizophrenia disorder spectrum and 20 educational level and age-matched healthy control. There are applied to every subjects different evaluation tests of the theory of mind: first and second order false-belief task; strange histories of Happe; faux pas test; the "reading the mind in the eyes» test; moral dilemma and face expressions recognition task. In addition the PANSS scale is applied to the group of patients, to value the psychopathological state at the moment of the evaluation. 
The results show significant differences between both groups in some of the applied tests, both in tests of cognitive processing and in tests in which an emotional processing of the information is necessary: Second order false-belief task; faux pas test; the "reading the eyes in the eyes» test and face expressions recognition task.

There are a lot of researches that centre how the deficits in this capacity determine the level of social adaptation. In case of the schizophrenia, the functional and social deficit might be explained largely by these problems in the attribution of mental and specially emotional states.

Koy words: SCHIZOPHRENIA; THEORY OF MIND; SOCIAL COGNITION; FIRST AND SECOND ORDER FALSE-BELIEF TASK; FAUX PAS TEST; STRANGE HISTORIES OF HAPPE;; THE RREADING THE MIND IN THE EYES, TEST; MORAL DILEMMA.

\section{INTRODUCCIÓN}

En las últimas décadas la esquizofrenia se ha considerado un trastorno del funcionamiento cerebral, por lo que han dominado las investigaciones sobre los determinantes biológicos de la enfermedad. Esta consideración se debe, en parte a los avances en los estudios de neuroimagen y neuropatologla, que aportan evidencias de alteraciones cerebrales en los pacientes esquizofrénicos y la aproximación a la etiologla desde los factores de riesgo genético. Los factores de riesgo genético son los únicos en los que se ha podido demostrar, según Martínez, Cuesta y Peralta (1997) un efecto suficiente como para considerarlos agentes causales de la esquizofrenia. Esto se reflejarla en una mayor presencia de trastornos esquizotípicos entre los progenitores de pacientes y un aumento del riesgo de padecer sintomas psicoticos en los pacientes con trastornos afectivos que tienen antecedentes familiares de esquizofrenia.

Los recientes avances de las técnicas de neuroimagen han permitido afirmar que los déficit cognitivos son una parte intrínseca de la enfermedad; asl los hallazgos más consistentes son el aumento de los ventrículos laterales, disminución del tejido cerebral, evidenciado 
en la mayor amplitud de los surcos corticales y la disminución de la sustancia gris y blanca (Wright, Rabe-Hesketh, Woodruff, David, Murray, y Bullmore 2000); reducción del volumen del hipocampo (Nelson, Saykin, Flashman y Riordan, 1998) y del tálamo (Konick y Friedman, 2001) y alteración del flujo sangulneo en la corteza prefrontal (Davidson y Heinrichs, 2003). Goldberg (2002) se centra en la disfunción del lóbulo frontal para describir las alteraciones cognitivas en la esquizofrenia. Se basa en la teoría de la afectación del sistema dopaminérgico mesolimbico-mesocortical como etiología de la enfermedad, sistema que se proyecta hacia el cortex prefrontal y que se ve alterado también en el síndrome disejecutivo tras un daño cerebral adquirido, sindrome que comparte alteraciones cognitivas con el trastomo esquizofrénico. El autor concluye por tanto que la esquizofrenia sería en gran medida una enfermedad del lóbulo frontal.

Según Muñoz Céspedes y Tirapu (2001), las alteraciones que han demostrado una relación consistente con la evolución a largo plazo de la enfermedad son: memoria, atención, funciones ejecutivas, lenguaje.

Alteraciones mnésicas: presentan recuerdo deficitario; la disfunción se asocia más a limitaciones de codificación que a problemas de almacenamiento o recuperación. La memoria de reconocimiento se halla escasamente alterada y la memoria procedimental se halla conservada.

Atención: limitación de los procesos atencionales; capacidad limitada para prestar atención a un estímulo relevante y discriminarlo de otros estímulos, siendo por tanto, más sensible a la distracción.

Funciones ejecutivas: que controlan la conducta mediante la regulación de las funciones de la atención, el manejo de las habilidades cognitivas para la planificación de la conducta, el desarrollo de comportamientos eficaces y socialmente aceptados y el empleo de la capacidad de abstracción. En la esquizofrenia estos déficit estarian relacionados con la capacidad para llevar a cabo tareas que requieran procesamiento complejo de información. Presentarian dificultades para formular planes de acción o para combinar las informaciones.

Lenguaje: el lenguaje en la esquizofrenia se hallaria intacto en cuanto a su léxico y sintaxis, pero existiría una incapacidad de estructurar el discurso a niveles superiores (en la utilización pragmática del discurso). El principal requisito para una comunicación adecuada es tener en 
cuenta el conocimiento, las creencias y las intenciones del interlocutor, aspectos que a menudo se ven afectados en el discurso de los pacientes.

A estas alteraciones ya descritas en los sujetos esquizofrénicos se ha venido a sumar en los últimos años la afectación de la denominada teorla de la mente. El origen del concepto se sitúa en 1978, cuando fue acuñado por vez primera por Premack y Woodruff, para referirse a la capacidad de atribuir estados mentales y predecir el comportamiento de otro organismo. Estos autores realizaron una serie de experimentos con chimpancés en los que se les mostraban imágenes de un cuidador encerrado en la jaula intentado alcanzar un plátano que estaba fuera de su alcance; la persona contaba con una serie de instrumentos con los que alcanzar el objetivo. En el momento en que el cuidador se disponla a intentar alcanzar el objeto, se congelaba la imagen y se mostraban al chimpancé dos fotografías, una de ellas con la solución correcta. Tras llevar a cabo varias sesiones experimentales, concluyeron que el simio era capaz de atribuir estados mentales como la intención y el conocimiento en otros, por lo que podrla afirmarse que estos primates tenlan «Teoria de la mente».

Tener una teorla de la mente implicarla, por tanto, ser capaz de atribuir estados mentales en uno mismo y en los demás con el fin de anticipar su comportamiento; de esta manera, debe entenderse como un mecanismo cognitivo, innatamente determinado, que permite un tipo especial de representación, como es la representación de los estados mentales (Premack y Woodruff, 1978). Se han formulado diversas definiciones sobre la teorla de la mente; quizás la más completa y que mejor recoge la complejidad del término serla «la habilidad para atribuir estados mentales independientes, como deseos, creencias y emociones, tanto en uno mismo como en otros" (Frith, 1992). Otros autores se centran en esas atribuciones y la utilidad de las mismas para hacer inferencias, interpretaciones y predicciones de las acciones propias y ajenas (Baron-Cohen, Wheelwrigth, Hill, Raste y Plumb, 2001; Premack y Woodruff, 1978). Desde la perspectiva evolucionista, la teoría de la mente surge en la evolución humana como respuesta a la complejidad cada vez mayor del entorno social. En esta linea surge la hipbtesis del cerebro social de Brothers (1990), que afirma que una buena capacidad metarrepresentacional puede relacionarse con un mayor éxito social. 
En sus inicios el concepto estuvo asociado a los trastornos generalizados del desarrollo y era utilizado como una hipótesis causal de la aparición de la enfermedad. Progresivamente comenzo a ser estudiado en otras patologlas como el daño cerebral adquirido (Bibby y McDonald, 2004 y Havet-Thomassin, Allain, Etcharry-Bouyx, Le Gall, 2006) las demencias (MacPherson, Phillips, Della Sala, 2002) y en los últimos años en la esquizofrenia (Mazza, de Risio, Sunian, Roncone, y Casacchia, 2001; Shamay-Tsoory, Aharon-Peretz y Levkovitz, 2007).

Existen hallazgos clínicos que sugieren que los pacientes con esquizofrenia presentarlan un déficit en la capacidad de interactuar socialmente, relacionado con su pobre capacidad comunicativa (Sperber y Wilson, 2002). Sin embargo existe controversia en torno a algunas cuestiones; por ejemplo si la teoria de la mente (capacidad de atribuir estados mentales en otros) sería deficitaria o estaria sobreactivada en la esquizofrenia. Otra cuestión que se plantea es si estas alteraciones representan un estado variable a lo largo de la evolución de la enfermedad o por el contrario se trata de un rasgo estable. Por último otra de las principales cuestiones que se han planteado es si los déficit en la esquizofrenia son específicos de la enfermedad, similares a los de los trastornos generalizados del desarrollo o si por el contrario son consecuencia de alteraciones cognitivas más generalizadas (atención, lenguaje, memoria, funciones ejecutivas). En torno a la última cuestión, existe evidencia a favor de la primera postura (Langdon, Coltheart, Ward y Catts, 2001); sin embargo, las alteraciones no serian similares a las observadas en el autismo. Aunque parece cierto que los déficit atencionales, el síndrome disejecutivo o una baja capacidad intelectual influyen negativamente en el rendimiento en tareas de teoría de la mente, las diferencias entre esquizofrénicos y sujetos sanos se mantienen cuando estas variables cognitivas son controladas.

Las líneas de investigación actuales toman los déficits en la teoría de la mente por un lado, como posibles causas de los síntomas positivos de la enfermedad (Tschacher y Kupper 2006). Mientras en otras investigaciones son tomados como variables mediadoras de las alteraciones en el funcionamiento social y adaptativo de los pacientes esquizofrénicos (Mazza, Costagliola, Di Michele, Magliani, Pollice, Ricci, Di Giovanbattista, Roncone, Casacchia y Galzio, 2007; Shamay- Tsoory, 
Shur, Barcai-Goodman, Medlovich, Harare y Levkovitz, 2006 y Bora, Eryavuz, Kayahan, Sungu y Veznedaroglu, 2006).

El presente estudio se centra en las enfermedades del espectro de la esquizofrenia. Para la evaluación de la teorla de la mente se han utilizado distintas pruebas con el fin de intentar recoger la complejidad del constructo evaluado, es decir, los componentes cognitivos y emocionales implicados.

\section{MATERIAL Y MÉTODOS}

La muestra consta de un total de 40 sujetos $(\mathrm{N}=40)$ divididos en dos grupos equiparados según características sociodemográficas (edad y nivel educativo). Por un lado un grupo de 20 pacientes de distintos dispositivos de la red de Salud Mental de Navarra, pertenecientes al Servicio Navarro de Salud Osasunbidea. Un grupo de 20 voluntarios sanos, sin antecedentes psiquiátricos y equiparados según edad y nivel educativo.

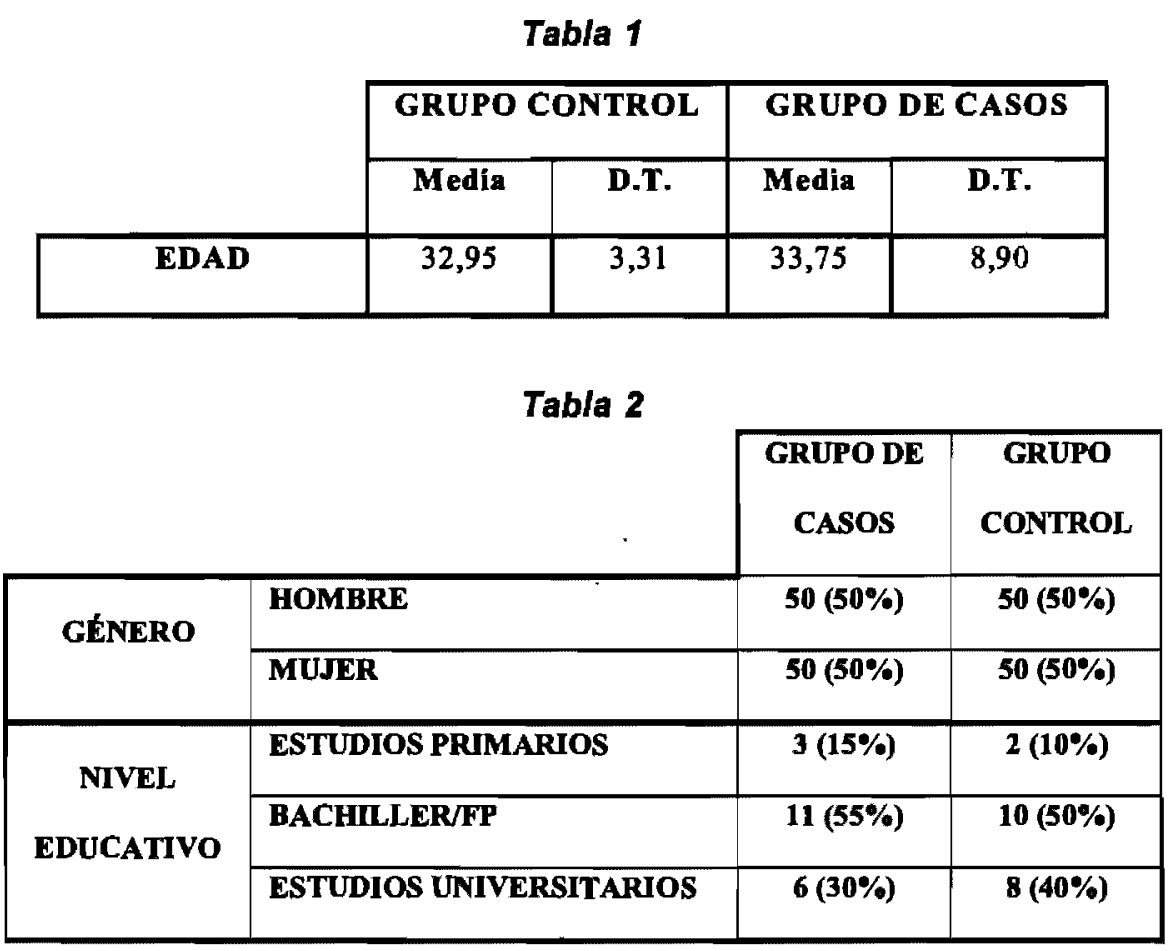


Todos los sujetos del grupo de casos han sido diagnosticados de esquizofrenia o trastorno esquizoafectivo (F20 esquizofrenia; F25 trastorno esquizoafectivo), según los criterios de la Clasificación Internacional de Enfermedades: ClE-10 (Organización Mundial de la Salud, 1992). De los 20 sujetos de la muestra 16 tienen diagnóstico de esquizofrenia ( $80 \%$ ); de éstos, 11 de tipo paranoide $(55 \%)$, 1 de tipo desorganizado (5\%), 1 de tipo residual $(5 \%)$ y 3 de tipo indiferenciado (15\%). Por otro lado 4 sujetos tienen diagnóstico de trastorno esquizoafectivo $(20 \%)$. Todos los sujetos reciben tratamiento neuroléptico. La media de ingresos en Unidad de Hospitalización Psiquiátrica es de $2(1,65)$, con un mínimo de 0 y un máximo de 6 hospitalizaciones. La edad media de inicio de la enfermedad se sitúa en 21,8 años $(7,56)$, y los años de evolución en la muestra tienen una media de $13,40(10,02)$.

Los datos sobre el diagnóstico, el número de ingresos psiquiátricos, la edad de inicio de la enfermedad y el tratamiento neuroléptico actual han sido recopilados de las historias clínicas de los pacientes.

Todos los pacientes reciben un consentimiento informado para la participación en el estudio, donde se les informa de los objetivos principales de los que consta el estudio y las diferentes pruebas que se les van a aplicar.

A todos los pacientes se les aplica la Escala de sintomas positivos y negativos PANSS. Por un lado se ha utilizado el sistema inclusivo para hacer una categorización de los pacientes entre síntomas positivos y síntomas negativos; asi 7 sujetos se incluirian en síntomas positivos $(35 \%)$ y 13 pacientes en síntomas negativos $(65 \%)$. Por otro lado se recogen las puntuaciones en cada una de las tres subescalas de la prueba (Escala Positiva; Escala Negativa y Escala de Psicopatología General), en función del rango en que se sitúa el percentil obtenido.

Para la evaluación de la teoría de la mente se han utilizado las siguientes pruebas:

1. Test de comprensión de creencias falsas (Wimmer y Perner, 1983)

Wimmer y Perner (1983) diseñaron una prueba, que se ha convertido en clásica para la evaluación de la teoría de la mente, pero además proporcionaron datos evolutivos determinantes sobre su 


\section{Tabla 3.}

\begin{tabular}{|c|c|c|c|c|}
\hline & & RANGO & SUJETOS & PORCENTANE \\
\hline \multirow{9}{*}{$\begin{array}{l}\frac{n}{5} \\
\frac{8}{8} \\
\text { 号 }\end{array}$} & \multirow{3}{*}{$\begin{array}{c}\text { Puntuación panss } \\
\text { positlva }\end{array}$} & 1 & 9 & $45 \%$ \\
\hline & & 2 & 8 & $40 \%$ \\
\hline & & 3 & 3 & $15 \%$ \\
\hline & \multirow{2}{*}{$\begin{array}{c}\text { Puntuación panss } \\
\text { negativa }\end{array}$} & 2 & 10 & $50 \%$ \\
\hline & & 3 & 10 & $50 \%$ \\
\hline & \multirow{4}{*}{$\begin{array}{c}\text { Puntuación } \\
\text { psicopatología } \\
\text { general }\end{array}$} & 1 & 9 & $45 \%$ \\
\hline & & 2 & 6 & $30 \%$ \\
\hline & & 3 & 4 & $20 \%$ \\
\hline & & 4 & 1 & $5 \%$ \\
\hline \multirow{2}{*}{ 送 } & $\begin{array}{c}\text { Sintomatología } \\
\text { positiva }\end{array}$ & & 7 & $35 \%$ \\
\hline & $\begin{array}{c}\text { SIntomatologia } \\
\text { negatlva }\end{array}$ & & 13 & $65 \%$ \\
\hline
\end{tabular}

Puntuaciones PANSS según rango $\rightarrow$ 1: muy bajo 2: bajo 3: medio 4: alto 5: muy alto

desarrollo y comenzaron una línea de trabajo sobre teorias explicativas que se ha ido desplegando desde la década de los 80 hasta la actualidad.

1.1. Creencias de primer orden: Esta tarea evalúa la habilidad de sujetos para inferir que alguien tiene una creencia (equivocada) que es distinta de la propia (verdadera) (Wimmer y Perner, 1983). Se basa en la localización inesperada de un objeto; el examinador describe una historia en la que el protagonista coloca un objeto (chocolate) en un lugar determinado (armario) antes de salir a jugar. En su ausencia, el objeto es cambiado de sitio (armario) y el sujeto no es informado de este traslado. A los sujetos se les pregunta dónde buscaría el objeto el protagonista cuando regrese a la escena inicial.

1.2. Creencia de segundo orden: Este tipo de tarea evalúa la capacidad de un individuo de comprender que alguien puede tener una falsa creencia acerca de la creencia o estado mental de un tercero. Nuevamente se le describe al sujeto una historia basada en la localización inesperada de un objeto; en ésta dos personajes son 
independientemente informados de la nueva localización inesperada de un objeto (furgoneta de helados); por tanto, ambos saben dónde está, pero hay un error en la creencia de segundo orden de un personaje sobre la creencia del otro, y a los sujetos se les pregunta sobre esta creencia errónea. Para poder responder las preguntas correctamente, el sujeto debe ser capaz de hacer una representación no solo la creencia de cada persona acerca de la ubicación, sino también de entender la falsa creencia de la persona segunda acerca de la creencia de la primera persona.

2. Test de las historias extranas (Happé, 1994)

Se han seleccionado algunas de las tareas de la bateria de «Historias extrañas», de Happe (1994a). De los 12 tipos de sentidos no literales representados en la bateria original, se han seleccionado 5 (mentira, mentira piadosa, persuasión, apariencia/ realidad y doble farol) que se encuentran traducidas en Happé (1994b). En las 5 historias los sujetos han de interpretar la intención o motivación del hablante. Por ejemplo, en la de «mentira piadosa» se trata de reconocer que se dice algo por no molestar a otros; en la de «persuasión» consiste en el intento de convencer a otro; en la de «doble farol» en engañar diciendo la verdad, pero haciendo creer a otros que se miente. Las intenciones o motivaciones no se extraen a partir del significado literal de lo que dice el personaje, sino que es preciso interpretarlas, para lo que es imprescindible basarse en las claves contextuales que proporciona la historia.

Las historias se narran a los sujetos y al finalizarlas se hace una pregunta: «¿por qué lo dice/hace?». Las respuestas dadas por el sujeto a esta pregunta se puntúan según el siguiente criterio: 0 si la respuesta es incorrecta; 1 si la respuesta es parcialmente correcta o expresada de forma implícita y 2 si la respuesta es correcta completa o expresada de forma explícita. Así la puntuación total de la prueba oscila entre un mínimo de 0 puntos a un máximo de 10.

3. Faux Pas Recognition Test (Baron-Cohen, O’Riordan, Stone, Jones y Plaisted, 1999)

Diseñado en sus inicios por Baron-Cohen et al. (1999) para niños de edades comprendidas entre 7 y 11 años. La tarea implica que el sujeto reconozca en una historia una «metedura de pata» de alguno de los protagonistas; situaciones en las que el protagonista dice algo 
sin considerar si es algo que el que escucha puede querer escuchar o saber y que típicamente conlleva consecuencias negativas que el que habla no tenla previstas. Si el sujeto detecta la metedura de pata, se le pide al sujeto que infiera la emoción del oyente al escuchar tal cosa. Si no lo hace se le realizan unas preguntas control para comprobar que ha entendido la historia. Según los autores, detectar una metedura de pata requiere entender las diferentes apreciaciones del hablante y del oyente, dependiendo del conocimiento de cada uno. Las historias incluidas en el test original fueron reunidas preguntando a personas por ejemplos de meteduras de patas de su propia experiencia. Posteriormente se presentó la lista a cuatro jueces, que tenian que evaluarlas como buenas historias, definiendo así la lista final de historias de las que consta la prueba.

En la aplicación cada historia se le presenta al sujeto y el administrador la lee en voz alta; después de leer la historia se le pregunta si cree que alguien en la historia ha dicho algo inadecuado o que no tenia que haber dicho. Según los propios autores las historias están redactadas en un lenguaje sencillo; la «metedura de pata» aparece en la anteúltima, penúltima o última frase para evitar que el sujeto utilice la estrategia de elegir siempre la última frase como respuesta. Si a la primera pregunta contestan que no se pasa directamente a las preguntas control y no se puntúan el resto de preguntas por lo que la puntuación total de la historia será 0 puntos. Si contesta sí se pasa a formular el resto de preguntas: ¿quién lo ha dicho?; ¿porqué no lo debería haber dicho?; ¿porqué crees que lo dijo?, ¿sabla $X$ que $Y$...? y ¿cómo se siente $Y$ ?. A cada una de estas preguntas contestadas correctamente se le otorga un punto; de tal forma que la puntuación mírima para cada historia es de 0 puntos y la máxima de 6. La puntuación total de la prueba se obtiene sumando las puntuaciones de cada historia, considerando la proporción de respuestas correctas sobre el máximo de aciertos posibles para cada uno de los ítems de la tarea. Las puntuaciones totales de la prueba oscilan entre 0 y 30 puntos.

4. Test de la rrirada, versión revisada (Baron- Cohen, Wheelwright, Hill, Raste y Plumb, 2001)

Es una prueba desarrollada por Barón-Cohen et al. en 2001 para la evaluación de la teoría de la mente en adultos. El test consta de 36 fotografias de ojos de hombres y mujeres que expresan un sentimiento 
- pensamiento. En cada fotografia aparece una mirada y cuatro palabras que hacen referencia a estados mentales o emocionales. EI sujeto debe elegir la palabra que considere que mejor describe lo que la persona de la fotografia está pensando o sintiendo. Le puede parecer que sea aplicable más de una palabra, pero deberá elegir aquella que considere la más apropiada. Debe intentar hacer la tarea lo más rápidamente posible, pero no se contabiliza el tiempo. Si no conoce el significado de alguna palabra puede pedir la definición exacta del manual de instrucciones.

Para la validación de la prueba pasaron el test a un grupo experimental: sujetos con síndrome de Asperger con un cociente intelectual normal, y dos grupos controles: adultos sanos con niveles educativos básicos, adultos sanos con estudios universitarios. Los resultados apuntaron a que tanto en los grupos experimentales como en los controles la ejecución del test de la mirada, correlacionaba inversamente con el cociente del espectro autista. En comparación con los grupos controles, el grupo experimental ejecutaba significativamente peor la tarea y no se daban diferencias significativas en la ejecución de los sujetos experimentales. Tampoco se encontraron diferencias significativas en cuanto a género en ninguno de los grupos del estudio.

El punto de corte se sitúa en 22 ( 22 aciertos del total de 36 fotografías presentadas), mientras que la puntuación media se sitúa en 26,2 (22 a 30).

5. Dilema moral del tren

Para abordar este nivel de la teoría de la mente se ha utilizado el Dilema del tren, recogido por Greene, Sommerville, Nystrom, Darley y Cohen, 2001, de la filosofia moral contemporánea. Estos autores se centran en el papel que juegan las emociones en razonamiento moral, alejándose de la psicología moral clásica de autores como Piaget y Kohlberg, que se centraban en el proceso puramente cognitivo implicado. Para ello se basan en los recientes avances en las neurociencias cognitivas y estudios de neuroimagen que recogen las áreas cerebrales que parecen estar implicadas en el razonamiento moral. Tanto las emociones como el razonamiento estarían implicados, pero el proceso emocional automático tiende a dominar el proceso.

El dilema del tren consta de dos partes; en la primera se expone la 
siguiente situación: «un tren se dirige sin control hacia un grupo de 5 operarios, que morirán si no se hace nada. Existe la posibilidad de apretar a un botón para provocar un cambio de agujas y que asl el tren se desvie a otra via dónde hay 1 operario trabajando, y éste morirla». Según Greene et al. (2001), la mayoría de las personas contestarian a esta pregunta que sI, argumentando que asi se salvarian más vidas. Posteriormente se plantea una versión del dilema «El sujeto se encuentra en un puente viendo la misma escena y la posibilidad de salvar a los 5 trabajadores es empujar a un hombre que hay al lado suyo que está tirado y parece haber bebido»; en este dilema la mayor parte de las personas responden que no le empujarian.

Para los autores, la diferencia entre ambos dilemas radica en el hecho de que en la segunda versión están implicadas las emociones; empujar a alguien y saber que va a morir es más emocionalmente saliente que apretar un botón, aunque en ambas situaciones las consecuencias sean las mismas. El procesamiento emocional en dilemas del segundo tipo tendría una mayor implicación, por lo que las áreas cerebrales asociadas con las emociones (corteza prefrontal ventromedial) estarlan más activas que en el primer dilema.

6. Reconocimiento de expresión facial de emociones básicas:

Se les presenta 24 vídeos de actores expresando una emoción con su cara y deben decidir de qué emoción se trata de entre las seis emociones básicas (Alegría; Tristeza; Sorpresa; Miedo; Asco e Ira). Se contabilizan los aciertos conseguidos en el primer intento.

Torras, Protell y Morgado (2001) han relacionado la amígdala con el procesamiento de las emociones y especialmente, con el reconocimiento de emociones representadas en las expresiones faciales. En este sentido, la lesión de la amigdala parece dificultar la capacidad para reconocer el significado afectivo de diferentes expresiones faciales, especialmente del miedo.

Para el análisis estadístico se ha utilizado el programa SPSS versión 11.5 para Windows.

\section{RESULTADOS}

En la línea de otras investigaciones (Shamay-Tsoory, Tomer, Berger, Goldsher y Aharon-Peretz, 2003; Leitman, Ziwich, Pasternak y Javitt, 
2006 y Russell, Rubia, Bullmore, Soni, Suckling, Brammer, Simmons, Williams y Sharma, 2000) en el presente estudio se han encontrado diferencias estadísticamente significativas entre ambos grupos en algunas de las pruebas: creencias de segundo orden; las tareas de Faux Pas; el test de la mirada y la prueba de reconocimiento de expresiones faciales.

\section{Tabla 4.}

\begin{tabular}{|c|c|c|c|c|}
\hline & & $\begin{array}{c}\text { GRUPO } \\
\text { DE } \\
\text { CASOS }\end{array}$ & $\begin{array}{c}\text { GRUPO } \\
\text { CONTROL }\end{array}$ & Chi $x^{2}$ \\
\hline \multirow{2}{*}{ Creencias $1^{\circ}$ orden } & 0 & $1(5 \%)$ & 0 & \multirow{2}{*}{0} \\
\hline & 1 & $19(95 \%)$ & $20(100 \%)$ & \\
\hline \multirow{2}{*}{ Creencias $2^{\circ}$ orden } & Algún error & $9(45 \%)$ & 0 & \multirow{2}{*}{$9,2^{\star \star}$} \\
\hline & Correcto & $11(55 \%)$ & $20(100 \%)$ & \\
\hline \multirow{2}{*}{$\begin{array}{l}\text { Historlas extrañas de } \\
\text { Happé }\end{array}$} & Algún error & $13(65 \%)$ & $8(40 \%)$ & \multirow{2}{*}{2,5} \\
\hline & Correcto & $7(35 \%)$ & $12(60 \%)$ & \\
\hline \multirow{2}{*}{ Faux Pas } & $<=23$ & $10(50 \%)$ & $2(10 \%)$ & \multirow{2}{*}{$7,6^{\star \star}$} \\
\hline & $>23$ & $10(50 \%)$ & $18(90 \%)$ & \\
\hline \multirow{2}{*}{ Dilema del tren } & Si/Si & $7(35 \%)$ & $4(20 \%)$ & \multirow{2}{*}{1,1} \\
\hline & Resto & $13(65 \%)$ & $16(80 \%)$ & \\
\hline \multirow{2}{*}{ Test de la mirada } & $<22$ & $14(70 \%)$ & $5(25 \%)$ & \multirow{2}{*}{$8,1^{\text {*\# }}$} \\
\hline & $>=22$ & $6(30 \%)$ & $15(75 \%)$ & \\
\hline \multirow{2}{*}{$\begin{array}{l}\text { Prueba de reconocimiento } \\
\text { de expresiones faciales }\end{array}$} & $<=19$ & $17(85 \%)$ & $5(25 \%)$ & \multirow{2}{*}{$14,5^{5}$} \\
\hline & $>19$ & $3(15 \%)$ & $15(75 \%)$ & \\
\hline
\end{tabular}

Nivel de significación

" $p<0,05$

$* p<0,01 \quad * * p<0,001$

Una de las pruebas donde se encuentran diferencias significativas entre ambos grupos es la prueba de creencias de segundo orden, que representaría el nivel más básico del constructo. Este resultado no serla concordante con lo sugerido por Shamay-Tsoory et al. (2006) sobre los déficit sociales en la esquizofrenia. Según estos autores los pacientes esquizofrénicos mostrarán más dificultades en tareas en las que sea necesario un procesamiento emocional de la información. 
Asi mientras que la tarea de creencias falsas (Wimmer y Perner, 1983) requiere la comprensión cognitiva de la diferencia entre el conocimiento del interlocutor y el del oyente (el conocimiento sobre creencia), captar la ironía en el test de las historias extrañas de Happé (1994) o las meteduras de pata en el test Faux Pas de Baron-Cohen et al., (1999) requieren empatizar con el estado emocional del oyente (conocer sus emociones). El grupo de Shamay-Tsoory sugiere que las tareas de inferencia de estados mentales cognitivos, y las tareas que implican la inferencia de estados afectivos tienen un deterioro diferencial en la esquizofrenia; los déficit en las competencias sociales estarian relacionados especificamente con la alteración de la faceta afectiva de la teoría de la mente.

Sin embargo si se han obtenido algunos datos a favor de la hipótesis de Shamay-Tsoory et al (2006); Así se han encontrado diferencias significativas entre ambos grupos en la ejecución del Test Faux Pas, en el que el sujeto debe empatizar con el interlocutor de la historia. También se observan diferencias en el Test de la mirada; según los autores del test (Baron- Cohen et al., 2001), el sujeto necesita poseer un léxico sobre estados mentales y conocer los significados semánticos de los términos, pero también implicaría que el sujeto abstraiga esos términos semánticos que se refieren a estados emocionales complejos y los asocie con sus expresiones faciales respectivas.

En recientes investigaciones se ha encontrado una asociación más sólida entre la sintomatologla negativa y las alteraciones en la teoría de la mente. Entre otras Mazza et al., 2007; Shamay-Tsoory et al., 2006; Bora et al., 2006. Otros estudios como el de Tschacher y Kupper (2006), obtienen resultados opuestos, ya que encontraron una mayor alteración de la percepción de causalidad, en estados de descompensación psicopatológica. En la muestra estudiada no se observa una asociación entre el tipo de sintomatología psicótica y la capacidad metarrepresentacional. Así no se han encontrado diferencias estadísticamente significativas entre ambos grupos de pacientes en ninguna de las pruebas aplicadas. 
Tabla 5.

\begin{tabular}{|l|l|c|c|c|}
\cline { 3 - 4 } \multicolumn{2}{c|}{} & $\begin{array}{r}\text { SINTOMAS } \\
\text { POSITIVOS }\end{array}$ & $\begin{array}{c}\text { SINTOMAS } \\
\text { NEGATIVOS }\end{array}$ & \multirow{2}{*}{ CHI X2 } \\
\hline \multirow{2}{*}{ Creencias de $1^{\circ}$ orden } & error & $1(14,29 \%)$ & 0 & \multirow{2}{*}{1,9} \\
\cline { 2 - 4 } & correcto & $6(85,71 \%)$ & $13(100 \%)$ & \\
\hline \multirow{2}{*}{ Creencias de $2^{\circ}$ orden } & error & $3(42,86 \%)$ & $6(46,15 \%)$ & \multirow{2}{*}{0} \\
\cline { 2 - 4 } & correcto & $4(57,14 \%)$ & $7(53,85 \%)$ & \\
\hline \multirow{2}{*}{$\begin{array}{l}\text { Historias extrañas de } \\
\text { Happé }\end{array}$} & error & $5(71,43 \%)$ & $8(61,54 \%)$ & \multirow{2}{*}{0,1} \\
\cline { 2 - 4 } & correcto & $2(28,57 \%)$ & $5(38,46 \%)$ & \\
\hline \multirow{2}{*}{ Faux Pas } & $<=23$ & $3(42,86 \%)$ & $7(53,85 \%)$ & \multirow{2}{*}{0,2} \\
\cline { 2 - 4 } & $>23$ & $4(57,14 \%)$ & $6(46,15 \%)$ & \\
\hline \multirow{2}{*}{ Test de la mirada } & $<22$ & $4(57,14 \%)$ & $10(79,92 \%)$ & \multirow{2}{*}{0,8} \\
\cline { 2 - 4 } & $>=22$ & $3(42,86 \%)$ & $3(23,08 \%)$ & \multirow{2}{*}{0,2} \\
\hline \multirow{2}{*}{ Dilema del tren } & incorrecto & $3(42,86 \%)$ & $4(30,77 \%)$ & \\
\cline { 2 - 4 } & correcto & $4(57,14 \%)$ & $9(69,23 \%)$ & \\
\hline \multirow{2}{*}{$\begin{array}{l}\text { Prueba de reconocimiento } \\
\text { de expresión facial }\end{array}$} & $<=19$ & $6(85,71 \%)$ & $11(84,61 \%)$ & \multirow{2}{*}{0} \\
\cline { 2 - 4 } & $>19$ & $1(14,29 \%)$ & $2(15,39 \%)$ & \\
\hline
\end{tabular}

Nivel de significación

"p<0,05" "p<0,01"**p<0,00

\section{DISCUSIÓN}

El constructo de teoría de la mente se engloba dentro de lo que se ha dado en llamar Cognición Social que incluiria, además de la teoria de la mente, otros campos del proceso cognitivo social como: procesamiento emocional, percepción social, conocimiento social y sesgos de atribución. Para Bar-On, Tranel, Denburg y Bechara (2003) incluiria la habilidad de reconocer y expresar emociones faciales; la habilidad de reconocer en otros sentimientos y establecer relaciones interpersonales; la habilidad de modular y regular emociones; la habilidad de darse cuenta, de forma realista y flexible de la situación y resolver problemas interpersonales y la habilidad de generar afectos positivos y automotivadores para conseguir los objetivos personales. Una adecuada competencia social implicaria entender las situaciones sociales y la información presente en tales situaciones. Para ello sería 
preciso según Mazza et al. (2007) un adecuado funcionamiento de la capacidad de mentalización o teorla de la mente, que permita entender e interpretar los estados mentales propios y ajenos, y hacer asi predicciones y explicaciones de las conductas.

Se podría concluir por tanto, que una capacidad de teorizar sobre estados mentales y emocionales es un componente necesario para un adecuado funcionamiento y adaptación social. Parece indiscutible el deterioro funcional y adaptativo en situaciones sociales con el que cursa la esquizofrenia, llegando a constituir un criterio diagnóstico en las Clasificaciones Internacionales: «Disfunción social/laboral: durante una parte significativa del tiempo desde el inicio de la alteración, una o más áreas importantes de actividad, como son el trabajo, las relaciones interpersonales o el cuidado de uno mismo, están claramente por debajo del nivel previo al inicio del trastomo ( 0 , cuando el inicio es en la infancia o adolescencia, fracaso en cuanto a alcanzar el nivel esperable de rendimiento interpersonal, académico o laboral)» (APA, 2003).

Como ya se ha comentado Shamay-Tsoory et al. (2006) plantean que los problemas de adaptación social en la esquizofrenia estarían relacionados especificamente con la alteración del componente afectivo de la teoria de la mente y de la carencia de empatía, más que con un deterioro general de las habilidades relacionadas. En esta línea Shamay-Tsoory et al. (2007), compararon el rendimiento en el reconocimiento de estados cognitivos y afectivos mediante tareas verbales y visuales en cuatro grupos: pacientes esquizofrénicos, pacientes con daño cerebral adquirido a nivel prefrontal ventromedial, pacientes con lesiones dorsolaterales y sujetos sanos. En los resultados se mostró que los pacientes con esquizofrenia y los pacientes con lesiones de la corteza prefrontal ventromedial tenían un peor rendimiento en las tareas de atribución de estados emocionales, en comparación con grupo control sano y los pacientes con lesiones prefrontales dorsolaterales; sin embargo no se encontraron estas diferencias en tareas cognitivas. Los autores concluyen que el déficit en la metarrepresentación de los esquizofrénicos es similar al de los sujetos con lesiones prefrontales ventromediales; añaden que estas similitudes apoyarian la hipótesis de una alteración de los circuitos fronto-límbicos en la esquizofrenia. 
En el presente estudio no sólo se han encontrado diferencias significativas en pruebas relacionadas con el componente emocional, sino también en aquellas en las que el sujeto debe hacer atribuciones sobre las creencias de otros (por ejemplo: creencias de segundo orden).

Brunet-Goudet y Decety (2006), realizan una revisión de los estudios de neuroimagen en los que se buscan las disfunciones cerebrales implicadas en el deterioro social de la esquizofrenia. Sugieren que una alteración en la transmisión dopaminérgica se asociaría a las alteraciones en las representaciones del contexto y a la persistencia de cogniciones falsas. Por otra parte, es posible que estas disfunciones crónicas o repetidas induzcan los cambios a largo plazo dando lugar a un círculo vicioso; asi la ansiedad y el malestar provocados por los pensamientos y opiniones anormales conducen a una falta de una motivación positiva para la vida social (aislamiento social). Los pacientes tienden a evitar la interacción social, reduciendo asi su entrenamiento social y disminuyendo su oportunidad de confrontar sus cogniciones anormales con puntos de vista objetivos; consecuentemente, disminuirían los recursos cognoscitivos para la solución de problemas interpersonales. En resumen una adecuada teoria de la mente podria considerarse como una variable predictiva del nivel de funcionamiento y adaptación social de los pacientes esquizofrénicos.

En esta línea, Bora et al. (2006) encontraron, como en el presente estudio, un peor rendimiento en el test de la mirada (Stone y BaronCohen, 1999) y que además éste era mejor predictor del funcionamiento social de estos sujetos, en comparación las capacidades cognitivas (p.e. Cociente Intelectual). Por ello concluyen que la capacidad de atribuir estados mentales y emocionales en otros (teoría de la mente), podría ser considerado como el procesamiento cognitivo más importante en el funcionamiento social. Los resultados obtenidos también muestran diferencias significativas en la ejecución del Test Faux Pas.

Para Tschacher y Kupper (2006), la percepción de causalidad sería otro factor implicado en la teoria de la mente y la cognición social en estos pacientes; sus creencias acerca de relaciones causa efecto, son a menudo erróneas y se desvian de las creencias de las personas sanas; asi en las ideas de referencia el paciente percibe relaciones 
causales entre él mismo y el entorno social, cuando en realidad esas relaciones no existen. Para Tschacher y Kupper los sintomas positivos están asociados a un incremento de la percepción de causalidad, mientras que los síntomas de desorganización estarian asociados a un descenso del factor. En la muestra del estudio no se encuentran diferencias significativas en la ejecución de los test en función de la sintomatología presente en el momento de la evaluación.

Por otro lado se han encontrado otros déficits de la teoria de la mente, por ejemplo en la capacidad de detectar significado de la prosodia del interlocutor, que también estaria directamente implicada en un adecuado funcionamiento social del individuo. Así Leitman, Ziwich, Parsternak y Javitt (2006), muestran un déficit significativo en la detección del sarcasmo, en comparación con los sujetos controles sanos. Mazza et al. (2007) de igual forma comparan pacientes psicóticos y neurológicos (lesiones prefrontales ventromediales derechas). También se muestra un perfil deficitario similar de ambos grupos en entender situaciones sociales y usar estrategias adaptativas para tales situaciones.

Otras estructuras cerebrales implicadas en el déficit del razonamiento social y la adaptación a estas situaciones en sujetos esquizofrénicos son el giro frontal izquierdo medio/inferior y la insula. Así Russell et al. (2000), mediante un estudio con resonancia magnética funcional, demuestran como los pacientes psicóticos tienen una menor activación en estas zonas, que los sujetos controles sanos, durante la ejecución del test de la mirada.

\section{REFERENCIAS BIBLIOGRÁFICAS}

American Psychiatry Association (2003). DSM-IV-TR: Manual diagnóstico y estadístico de los trastornos mentales. Barcelona. MASSON

Bar-On, R., Tranel, D., Deriburg, N. \& Bechara, A. (2003) Exploring the neuroanatomical substrate of emotional and social intelligence. Brain, 126, 1790-1800

Baron-Cohen, S., O'Riordan, M., Stone, V., Jones, R. \& Plaisted, K (1999). Recognition of Faux Pas by normally developing children and children with Asperger Syndrome or High-functioning autism. Journal of Autism and Developmental Disorers, 29 (5), 407-418. 
Baron-Cohen, S., Wheelwrigth, S., Hill, J., Raste, Y., \& Plumb, I. (2001). The ureading the mind in the eyes» test revised version: a study with normal adults and adults with Asperger Syndrome or high-functioning autism. Joumal of Child Psychology and Psychiatry, 42 (2), 241-251 Bibby, H. \& McDonald, S. (2004). Theory of mind after traumatic brain injury. Neuropsychology, 43 (1), 99-114.

Bora, E.; Eryavuz, A.; Kayahan, B.; Sungu, G. \& Veznedaroglu, B. (2006). Social functioning, theory of mind and neurocognition in outpatients with schizophrenia; mental state decoding may be a better predictor of social functioning than mental state reasoning. Psychiatry Research, 145, 95-103.

Brothers, L. (1990). The social brain: a project for integrating primate behavior and neurophysiology in a new domain. Concepts in Neuroscience, 1, 27-51.

Brunet-Gouet, E. \& Decety, J. (2006). Social brain dysfunctions in schizophrenia: a review of neuroimaging studies. Psychiatry Research: Neuroimaging, 148, 75-92.

Davidson, L.L. \& Heinrichs, R.W. (2003). Quantification of frontal and temporal lobe brain-imaging findings in schizophrenia: a metaanalysis. Psychiatry Research, 122, 69-87.

Frith, C.D. (1992). The cognitive neuropsychology of schizophrenia. Hove: Lawrence Erlbaum Associates.

Greene, J., Sommerville, B., Nystrom, L., Darley, J. \& Cohen, J. (2001). An FMRI investigation of emotional engagement in moral judgment. Science, 293, 2105-2108

Goldberg, E. (2002). El cerebro ejecutivo. Madrid: Critica.

Happé, F. (1994a). An advanced test of theory of mind: understanding of store characters' thoughts and feelings by able autistic, mentally handicapped, and normal children and adults. Journal of Autism and Developmental Disorders, 24 (2), 129-154.

Happé, F. (1994b). Introducción al autismo. Madrid alianza editorial (traducción de Maria Nufiez, 1998)

Havet-Thomassin, V., Allain, P., Etcharry-Bouyx, F. \& Le Gall, D. (2006). What about theory of mind after severe brain injury?. Brain Injury, 20(1), 83-91.

Konick, L.C. \& Friedman, L. (2001). Meta-analysis of thalamic size in schizophrenia. Biological Psychiatry, 49, 28-38. 
Langdon, R., Coltheart, M., Ward, P.B. \& Catts, S.V. (2001). Mentalising, executive planning and disengagement in schizophrenia. Cognitive Neuropsychiatry, 6, 81-108.

Leitman DI,. Ziwich R., Pasternak R. \& Javitt DC. (2006). Theory of Mind (ToM) and counterfactuality deficits in schizophrenia: misperception or misinterpretation? Psychological Medicine, 36 (8), 1075-1083.

Macpherson, S.E., Phillips, L.H. \& Della Sala, S. (2002). Age, executive function, and social decision making: a dorsolateral prefrontal theory of cognitive aging. Psychology and Aging, 17 (4), 598-609 Martinez, A., Cuesta, M.J. \& Peralta, V. (1997). Etiologia de la Esquizofrenia: revisión de la interacción genes-ambiente. Anales del Sistema Sanitario de Navarra, 20 (2), 183-189.

Mazza, M.D., de Risio, A.; Surian, L.; Roncone, R. \& Casacchia, M. (2001). Selective impairments of theory of mind in people with schizophrenia. Schizophrenia Research, 47, 299-308.

Mazza M.D., Costagliola C., Di Michele V., Magliani V., Pollice R., Ricci A., Di Giovanbattista E., Roncone R., Casacchia M., \& Galzio RJ. (2007). Deficit of social cognition in subjects with surgically treated frontal lobe lesions and in subjects affected by schizophrenia. European Archives of Psychiatry Clinical Neuroscience, 257 (1), 12-22

Muñoz Céspedes, J. M., \& Tirapu Ustárroz, J. (2001). Rehabilitación neuropsicológica. Madrid: Síntesis.

Nelson, M.D., Saykin, A.J, Flashman, L.A. \& Riordan, H.J. (1998). Hippocampal volume reduction in schizophrenia as assessed by magnetic resonance imaging: a meta-analytic study. Archives of General Psychiatry, 55, 433-440.

Peralta V. \& Cuesta MJ. (1994). Validación de la escala de los síndromes positivo y negativo (PANSS) en una muestra de esquizofrénicos españoles. Actas Luso-Españolas de Neurología y Psiquiatría 22 (4), 171-177.

Premack, D. \& Woodruff, G. (1978). Does the chimpanzee have a theory of mind? Behavioral and Brain Sciences, 4, 512-526.

Russell, T.; Rubia, K.; Bullmore, E.; Soni, W.; Suckling, J.; Brammer, M.; Simmons, A.; Williams, S. \& Sharma T. (2000). Exploring the Social Brain in Schizophrenia: Left Prefrontal Underactivation During 
Mental State Attribution. American Journal of Psychiatry, 157, 20402042

Shamay-Tsoory, SG., Tomer, R., Berger, BD., Goldsher, D. \& AharonPretz, J. (2003). Impairment affective theory of mind is associated with right ventromedial prefrontal damage. Cognitive and behaviour neurology, 18 (1), 55-67

Shamay-Tsoory SG., Shur S., Barcai-Goodman L., Medlovich S., Harari H., \& Levkovitz Y. (2006). Dissociation of cognitive from affective components of theory of mind in schizophrenia. Psychiatry Research, 13; 11-23

Shamay-Tsoory SG., Aharon-Peretz J. \& Levkovitz Y. (2007). The neuroanatomical basis of affective mentalizing in schizophrenia: Comparison of patients with schizophrenia and patients with localized prefrontal lesions. Schizophrenia Research, 90 (1-3), 274283.

Sperber, D. \& Wilson, D. (2002). Pragmatics, modularity and mindreading. Mind and Language, 17, 3-23.

Torras, M., Portell, I. \& Morgado, I. (2001). La amigdala implicaciones funcionales. Revista de Neurología, 33 (5), 471-476

Tschacher W., \& Kupper Z. (2006). Perception of causality in schizophrenia spectrum disorder. Schizophrenia Bulletin, 32 Suppl 1,106-112.

Wimmer, H. \& Perner, J. (1983). Beliefs about beliefs: representation and constraining function of wrong beliefs in young children's understanding of deception. Cognition, 13 (1), 103-128

Wright, I.C., Rabe-Hesketh, S., Woodruff, P.W., David, A.S., Murray, R.M. \& Bullmore, E.T. (2000). Meta-analysis of regional brain volumes in schizophrenia. American Journal of Psychiatry, 157, 16-25. 